\title{
EL MINISTERIO PÚBLICO Y LA POLÍTICA CRIMINAL EN UNA SOCIEDAD DEMOCRÁTICA
}

\author{
Jean Pierre Matus Acuña
}

\begin{abstract}
RESUMEN
En el artículo se establecen los parámetros de lo que se estima una adecuada política criminal para el Ministerio Público, basada en metas de gestión que clarifiquen su rol como agente en la reducción de las tasas de criminalidad al aumentar mediante sus actuaciones el costo de la comisión de delitos (según el modelo que Gary Becker recoge de Beccaria y Bentham), haciéndose, de paso, un juicio crítico a las propuestas actuales de la institución en la materia.
\end{abstract}

\section{MINISTERIO PÚBLICO - POLÍTICA CRIMINAL - COSTO DEL DELITO}

\section{The prosecutoring authority and criminal policies in a democracy}

\begin{abstract}
This article discuss criteria for appropriate criminal policies in relation to the prosecutoring authority, based on management goals that clarify its role as an agent in reducing the rates of crime by increasing, through its actions, the cost of committing crimes (according to the model that Gary Becker took from Beccaria and Bentham). On the way, it makes a critical judgment of the actual proposals of the institution in this regard.
\end{abstract}

\section{PROSECUTORING AUTHORITY-CRIMINAL POLICIES - THE COST OF CRIME}

* Abogado, Doctor en Derecho, Profesor Asociado de la Universidad de Talca, Director Centro de Estudios de Derecho Penal, Campus Santiago Universidad de Talca, Chile. Correo electrónico: jpmatusa@ utalca.cl, Artículo recibido el 28 de abril de 2006 y aceptado para su publicación por el Comité Editorial el 30 de mayo de 2006. 


\section{INTRODUCCIÓN}

$\mathrm{E}$ n su última Cuenta Pública, el Fiscal Nacional del Ministerio Público ha declarado que al modificar la Ley 20.074 el artículo 21 de la ley 19.640, Orgánica del Ministerio Público, estableciendo que el Fiscal Nacional en su cuenta pública podrá "sugerir las políticas públicas y modificaciones legales que estime necesarias para el mejoramiento del sistema penal, para una efectiva persecución de los delitos, la protección de las víctimas y de los testigos y el adecuado resguardo del derecho de las personas", se "viene a recoger una de las recomendaciones formuladas por la Comisión de Expertos, que en el año 2003 sugirió diversos ajustes a la reforma procesal penal", lo que "importa un explícito reconocimiento a la institución Ministerio Público [como el] órgano estatal más capacitado y experimentado en la problemática penal”, agregando que, "si bien el Ministerio Público elabora y ejecuta políticas propias de persecución penal, su misión hoy debe complementarse mediante propuestas que mejoren la actividad estatal de otros sujetos que intervienen en el proceso penal". ${ }^{1}$

En este artículo se expondrá una visión de lo que esas políticas públicas, entendidas como política criminal, debieran comprender ${ }^{2}$ y una crítica, sobre la base de ese entendimiento, a las propuestas realizadas por el Ministerio Público al respecto.

\section{La Política Criminal y el Ministerio Público}

En los documentos preparatorios a la citada Séptima Cuenta Pública del Ministerio Público, y en contraste con la anterior posición oficial del Fiscal Nacional en materia de seguridad ciudadana, ${ }^{3}$ la Comisión de Políticas Criminales del Ministerio Público estimaba que la facultad entregada al Fiscal Nacional en el nuevo inciso final del Art. 21 de ley Orgánica del Ministerio Público, le otorga una "oportunidad histórica" de "hacer una propuesta sobre delincuencia, prevención y represión del delito, sugiriendo no sólo las áreas prioritarias, sino que también los medios idóneos, entre los cuales cabe destacar las modificaciones legales de carácter penal y/o procesal penal que se estimen

${ }^{1}$ Piedrabuena, G., "Séptima Cuenta Pública del Ministerio Público, Efectuada por el Fiscal Nacional, Dn. Guillermo Piedrabuena (Art. 21 de la Ley 19.640), 28 de abril de 2006”, en http://www.ministeriopublico.cl/index.asp, visitado el 20 de mayo de 2006, p. 47.

${ }^{2}$ Una versión preliminar de esta visión se leyó en Santiago, el día 24 de enero de 2006, ante la Comisión de Políticas Criminales del Ministerio Público, por invitación del Sr. Fiscal Nacional.

${ }^{3}$ Cfr. Piedrabuena, G., "Quinta Cuenta Pública de Actividades del Ministerio Público, Efectuada por el Fiscal Nacional, Don Guillermo Piedrabuena Richard (Art. 21 de la Ley 19.640), de 26 de abril de 2004”, en http://www.ministeriopublico.cl/index.asp, visitada el 10 de enero de 2006, p. 34s. Allí se señala que: "El actual marco legal asigna al Ministerio Público la función de dirección de la investigación, de brindar protección a las víctimas y testigos y la labor de sostener la acción penal pública, no así funciones cuyo ejercicio es previo a la comisión de un delito, como es el caso de las funciones ligadas a la seguridad ciudadana, que poseen un tinte claramente preventivo. Por lo anterior, el Ministerio Público no cuenta con facultades legales para hacerse cargo de este último tema". 
necesarias para la obtención de los resultados propuestos", esto es, una propuesta de política criminal, pero haciendo hincapié en el carácter técnico o neutro que debieran tener tales propuestas. ${ }^{4}$

En este concepto parecía asumirse implícitamente que los hechos constitutivos de delito son males que una sociedad democrática desea evitar, previniendo su comisión y reprimiendo a los responsables, con pleno respeto a las garantías constitucionales. Luego, uno podría suponer que el objetivo general de esas propuestas de políticas públicas sería, naturalmente, reducir esos males, esto es, proponerse lograr una disminución significativa en la tasa de delitos que se cometen. ${ }^{5}$ Y que su carácter "neutro" o "técnico" radicaría en que la determinación de esos males que se pretenden evitar, esto es, de los hechos constitutivos de delito, sería competencia exclusiva de los poderes políticos que la Constitución establece.

Pero como no tienen relevancia aquí las medidas de prevención ajenas a las que puedan adoptar las personas que forman parte de las instituciones que componen el sistema penal, el objetivo general enunciado puede concretarse diciendo que se trataría de establecer metas de reducción de la actividad criminal en áreas prioritarias (p. ej., los llamados delitos de mayor connotación social), en lugares (comuna, región, Estado) y tiempos (meses o años) determinados, explicitando las actividades a realizar y los medios que cada institución del sistema penal (Ministerio Público, policías, jueces, Gendarmería de Chile, Sename) debe emplear para lograrlas.

De allí resulta que las políticas públicas en la materia forman una matriz donde la fijación de metas, prioridades, actividades y medios a nivel institucional debe desplegarse territorial y temporalmente, esto es, a nivel local, regional y nacional; y el corto, mediano y largo plazo, respectivamente.

Esta matriz es similar a la que se utiliza en elaboración e implementación de los Planes Regionales Anti Delincuencia, y por lo mismo les puede parecer una obviedad. Sin embargo, es útil pues permite poner en primer plano de la discusión algo que es, a mi juicio, esencial para que el Fiscal Nacional pueda legítimamente sugerir a los colegisladores y al resto de las instituciones del sistema penal reformas legales procesales y penales y otras medidas de políticas públicas de prevención y represión del delito: fijar en su propia política de persecución criminal metas de reducción de la criminalidad, estableciendo la clase de delitos cuya incidencia estima prioritario reducir, el quántum de reducción efectiva que espera lograr, qué medidas se adoptarán local, regional o nacionalmente, cuáles medios han de emplearse en ello, y cuándo se espera conseguir los resultados propuestos. A falta de una política criminal propia explícita, la labor de sugerir a otras instituciones políticas públicas en materia de prevención y represión del delito carecerá de un punto de partida sólido y va a estar sujeta a una seria y muy fundada crítica.

\footnotetext{
${ }^{4}$ Comisión de Política Criminal del Ministerio Público, Minuta Sobre el alcance de la modificación introducida por la Ley 20.074 al Art. 21 de la Ley Orgánica del Ministerio Público, Noviembre de 2005, no publicada, p. 8.

${ }^{5}$ Para la discusión sobre el valor de las tasas de denuncias como indicador de la actividad criminal, Cfr. Matus, J. P.: “¿Por qué no bajan las tasas de criminalidad en Chile?”, en Revista de Derecho de la Pontificia Universidad Católica de Valparaíso, vol. XXVI (2005), Nº 1, pp. 67-92.
} 
A continuación, se expondrá (I) la actual política criminal del Ministerio Público, demostrando de paso que ella no ha sido suficientemente explicitada y que no tiene como objetivo directo la reducción de la criminalidad; (II) cómo dicha política eventualmente podría influir en la prevención del delito; (III) qué límites me parecen existen a la facultad otorgada al Fiscal Nacional de sugerir políticas públicas de prevención y represión del delito a otras instituciones; y (IV) un análisis crítico de las medidas propuestas en la materia para el año 2006 por el Ministerio Público.

\section{LA ACTUAL POLÍTICA CRIMINAL (POLÍTICA DE PERSECUCIÓN PENAL) del Ministerio Público no está explicitada, y no tiene COMO META DE REDUCIR LA CRIMINALIDAD}

En la idea de que a la política criminal corresponde "determinar los bienes jurídicos que deben protegerse y la forma como materializar dicha protección”, ${ }^{6}$ tenemos dos funciones claramente diferenciadas: por una parte, "determinar los bienes jurídicos que deben protegerse”, estableciendo en la Ley qué hechos se considerarán constitutivos de delito, labor entregada al legislador; y, por otra, determinar "la forma de materializar dicha protección”, función entregada parcialmente al Ministerio Público, en tanto órgano autónomo encargado de la investigación criminal y el sostenimiento de la acción penal. Como estas atribuciones se ejercen tomando decisiones sobre en qué condiciones archivar o no los procedimientos, en qué casos otorgar a los imputados el principio de oportunidad, cuándo correspondería negociar una suspensión condicional del procedimiento, cuándo acceder a un procedimiento abreviado o uno simplificado, y cuándo seguir con el juicio oral hasta el final, en los hechos, todos los días y todos los fiscales adjuntos del Ministerio Público están tomando decisiones de política criminal, esto es, decisiones que hacen realidad la política de persecución penal del Ministerio Público y que suponen la existencia de criterios sobre cuáles delitos se estima prioritario perseguir, "con todo el rigor de la ley”, y cuáles no; en qué casos puede estimarse que un imputado no volverá a delinquir de llegarse a una salida alternativa y qué casos no; cuánto tiempo y recursos invertirá la Fiscalía en la investigación y sostenimiento ante los tribunales del caso o la policía en cumplir sus instrucciones; cuánta inversión en protección de víctimas y testigos es adecuada a la investigación en curso, etc.

Por las reglas que permiten a los Fiscales Regionales controlar ciertas decisiones estratégicas de los fiscales adjuntos y darles instrucciones particulares; reservando para el Fiscal Nacional sólo la posibilidad de dar instrucciones de carácter general, podemos asumir que existen dos niveles claves en la determinación de la política de persecución criminal del Ministerio Público: el nacional y el regional. ${ }^{7}$ La puesta en marcha de

\footnotetext{
${ }^{6}$ Comisión, op. cit., p. 7.

${ }^{7}$ Sin embargo, según como cada Fiscal Regional ejerza sus atribuciones y entienda la supuesta "autonomía” de los fiscales adjuntos, es evidente que pueden surgir políticas de persecución penal locales, sobre
} 
comisiones institucionales para hacer operativo el nuevo artículo 17a) de la LOCMP que, para los delitos "que generen mayor connnotación social”, ha previsto dictar instrucciones generales diferenciadas por regiones en materias de salidas alternativas y diligencias inmediatas, puede ayudar a evitar la dispersión de criterios, pero el hecho de que se encuentren separadas de esta Comisión de Políticas Criminales podría originar decisiones contradictorias, que deben prevenirse, puesto que entre los objetivos que señala el artículo 17a) deben tener tales instrucciones no se encuentra la reducción de la actividad criminal, sino sólo "los señalados en la Constitución y las leyes", que no son objetivos, sino funciones.

Antes de estas últimas modificaciones legales, el Fiscal Nacional, a través de sus Instrucciones Generales y Oficios ya había establecido (sin declararlo explícitamente) la política nacional de persecución penal del Ministerio Público, cuyo sello actual es "moderada maximización punitiva", en el sentido de desestimular los "términos anticipados" y las llamadas "salidas alternativas" a favor de la obtención de sentencias como forma preferente de término, particularmente respecto de los delitos calificados por los sistemas de internacionales adoptados por el Gobierno como de mayor connotación social (especialmente, hurtos, lesiones, robos, homicidios y delitos sexuales), muchos de los cuales equívocamente se califican, al interior del Ministerio Público, como "delitos menores". ${ }^{8}$ A esta política nacional de persecución penal se superponen las políticas que cada Fiscal Regional adopta en las metas de desempeño que imponen a sus fiscales adjuntos, y en otras decisiones más sutiles y, por el momento, difíciles de explicitar, como la forma de organización interna de cada Fiscalía Regional, los recursos que se destinan a determinadas fiscalías locales, las funciones “especiales” que eventualmente se les asignan, y los premios y castigos que se distribuyen en las evaluaciones anuales de desempeño.

Pero, la reducción de la actividad delictiva no se encuentra entre los objetivos actuales de la política de persecución criminal del Ministerio Público y, por tanto,

todo en las Regiones donde las distancias favorecen la toma de decisión sin control. Pero no podemos entrar ahora a analizar la existencia de estas políticas locales de persecución criminal.

${ }^{8}$ Cfr. Matus, J.P.: "Criterios de actuación del Ministerio Público en materias penales. Recensión del texto 'Reforma procesal penal. Oficios del Fiscal Nacional. Materias penales'. 2001-2004. Santiago 2005”, en Ius et Praxis, vol 11 (2005) No 2, pp. 357ss. Sus aspectos distintivos son:

a) Menor discrecionalidad para decretar el archivo provisional, obligando aun en casos de los equívocamente denominados "delitos menores", decretar una orden de investigar (viable o no) y una revisión global y periódica de los casos archivados (Oficio $\mathrm{N}^{\circ}$ 096);

b) Reducción de los casos en que es posible ejercer el principio de oportunidad, i.e., en el delito de receptación (Oficio $\mathrm{N}^{\circ} 258$ ) y todo aquél en que haya participado un funcionario público, sea o no delito funcionario (Oficio $\mathrm{N}^{\circ} 551$ ); imposición de exigencias especiales para ejercerlo, como considerar "el interés concreto de la víctima” en los hurtos (Oficio $\mathrm{N}^{\circ} 258$ ), o acuerdo escrito de ésta en los delitos contra la propiedad intelectual (Oficio $\mathrm{N}^{\circ} 150$ );

c) Desvalorización de la suspensión condicional del procedimiento, considerándola "no recomendable" en los delitos contra la propiedad intelectual (Oficio $\mathrm{N}^{\circ} 150$ ), "no aplicable" en los funcionarios (Oficio $\mathrm{N}^{\circ} 551$ ), en los sexuales donde son víctimas menores ( $\mathrm{N}^{\circ}$ 096) y, en general, ordenando a instar por ella "de manera reflexiva y selectiva" ( $\left.\mathrm{N}^{\circ} 096\right)$

d) Preferencia por el procedimiento ordinario para evitar sorpresas derivadas de las facultades judiciales en los procedimientos abreviado y simplificado ( $\mathrm{N}^{\circ}$ 096). 
la eficacia en su gestión no se mide con relación a este parámetro. En realidad, según los énfasis que pueden verse en la Cuenta Pública del Fiscal Nacional de 2005, hasta el momento parece que el objetivo principal del Ministerio Público, a nivel nacional, ha sido dar pronto término a los casos, aumentando al mismo tiempo el porcentaje de "términos judiciales", incluyendo un aumento en los juicios orales. ${ }^{9}$ Este énfasis repercute en la competencia entre las diferentes Fiscalías Regionales por obtener los mejores resultados conforme a esos indicadores y así estar en mejor pie para obtener los recursos adicionales necesarios para profundizar los resultados obtenidos, como se trasluce de las informaciones de prensa que recogen las cuentas públicas de las Fiscalías Regionales de la Región Metropolitana del año 2006. ${ }^{10}$

\section{INFLUENCIA DE LA POLÍTICA DE PERSECUCIÓN CRIMINAL DEL MiNISTERIO PÚBLICO EN LA PREVENCIÓN DEL DELITO: DISUASIÓN E INCAPACITACIÓN}

Es casi un lugar común afirmar que quienes están de alguna manera concernidos en la administración de políticas públicas vinculadas a la actividad criminal son más propensos a suponer que su aumento se debe a factores sociales o personales incontrolables y, al contrario, atribuirse como logro de su propia gestión el descenso en las tasas de delitos. ${ }^{11}$ Sin embargo, a pesar de que los factores a que se atribuye el aumento y la disminución de la actividad criminal son múltiples y de diferente origen, no son tantos como para renunciar a su estudio, ni tan ajenos a los estímulos creados por la propia comunidad que no existan ciertas posibilidades de control. La lista es corta y, sin duda, bastante estudiada (aunque no en Chile), ${ }^{12}$ e incluye: la situación económica nacional, ${ }^{13}$ las regulaciones sobre control de armas, ${ }^{14}$ la cantidad de policías por cada cien mil habitantes, ${ }^{15}$ las políticas de gestión policial tipo "tolerancia cero" y "policías

${ }^{9}$ Piedrabuena, G., "Sexta Cuenta Pública de Actividades del Ministerio Público, Efectuada por el Fiscal Nacional, don Guillermo Piedrabuena Richard (Art. 21 de la Ley 19.640), de 26 de abril de 2005", en http://www.ministeriopublico.cl/index.asp, visitado el 2 de mayo de 2006, p. 8s.

${ }^{10}$ Cfr., El Mercurio, Cuerpo C, Sábado 21 de enero de 2006.

${ }^{11}$ Blumstein, A. y Rosenfeld, R.: "Explaining Recent Trends in U.S. Homicide Rates”, en Journal of Criminal Law and Criminology, vol. 88 (1998), No 4, p. 1179.

${ }^{12}$ Levitt, S.: "Understanding Why Crime Fell in the 1990s: Four Factors that Explain the Decline and Six that Do Not", en Journal of Economics Perspectives, vol. 18 (2004), No 1, pp. 163-190. Su traducción puede verse en Ius et Praxis, vol. 10 (2004) No 2, pp. 97-137, traductor: Matus, J. P.

${ }^{13}$ Cfr. Núñez, J., Rivera, J., Villavicencio, X. y Molina, Ó.: “Determinantes socioeconómicos y demográficos del crimen en Chile: evidencia desde un panel de datos de las regiones chilenas”, en Estudios de Economía, vol. 30 (2003), No 1, pp. 55-85.

${ }^{14}$ Cfr. Kennedy, D. et al: "Youth Violence in Boston: Gun Markets, Serious Youth offenders, and a Use-reduction strategy", en Law and Contemporary Problems, vol. 59 (1996), No 1, pp. 147-195, y especialmente, Lott, J. y Mustard, D.: "Right-to-Carry Concealed Guns and the Importance of Deterrence", en Journal of Legal Studies, vol. 26 (1997), Enero, pp. 1-68.

${ }^{15}$ Cfr. Marvell, T. y Moody, C.: “Specification Problems, Police Levels, and Crime Rates”, en Criminology, vol. 34 (1996.), Noviembre, pp. 609-46. 
comunitarios", ${ }^{16}$ el aumento del tiempo de duración de las penas y de su gravedad, ${ }^{17}$ el número de personas en prisión, ${ }^{18}$ los cambios demográficos, ${ }^{19}$ los cambios en el mercado de las drogas ilícitas ${ }^{20}$ y las tasas de hijos no deseados. ${ }^{21}$

De esa lista de factores, es evidente que se encuentra fuera del campo de acción del Ministerio Público adoptar políticas económicas, sociales y demográficas, aunque bien puede destinar recursos al estudio de su influencia en la actividad criminal. Tampoco le corresponde modificar la regulación legal del control de armas y, en general, de los delitos y las penas que se les asignan.

En cambio, aunque el Ministerio Público no puede fijar las plantas policiales, su propia política de persecución penal sí influye en el factor relativo al número de policías disponibles y a la gestión de su actividad: puesto que el Ministerio Público utiliza recursos policiales en sus investigaciones y en la presentación de sus casos ante los tribunales, sería deseable que pudieran cuantificarse esos recursos (especialmente las horas-hombres), para poder determinar: a) la eficiencia de su uso en el éxito de las investigaciones; y b) el costo alternativo (en términos de reducción de las tasas de delitos) que ello tiene con relación a su distracción de las funciones de prevención situacional y del llamado "gestionamiento policial" (patrullajes callejeros, relaciones con la comunidad, vigilancia focalizada, etc.).

Pero donde más claramente parece que de alguna manera la actuación del Ministerio Público refleja la afirmación contenida en un documento de su Comisión de Políticas Criminales, según la cual, "en la actualidad, la separación entre prevención y represión del delito se ha diluido bastante", ${ }^{22}$ es en relación con los factores vinculados a los tiempos de duración de las condenas y el número de personas cumpliendo penas privativas de libertad. En efecto, según el modelo dominante en la criminología basada en estudios econométricos, al menos respecto de los delitos de motivación pecuniaria (hurtos y robos), que conforman el grueso de las estadísticas de los denominados "de mayor connotación social”, los resultados de la forma cómo el Ministerio Público sostiene la acción penal deben considerarse para cuantificar el costo relativo de la comisión

\footnotetext{
${ }^{16}$ Cfr. De la Puente, P. y Torres, E.: "Seguridad ciudadana y prevención del delito. Un análisis crítico de los modelos y estrategias contra la criminalidad”, en Estudios Criminológicos y Penitenciarios, $\mathbf{N}^{\circ} 1$ (2000), pp. 15-62.

${ }^{17}$ Cfr. Langan, P.: “America's Soaring Prison Population”, en Science 251 (1991), marzo, pp. $1568-1573$.

${ }^{18}$ Cfr. Marvell, T. y Moody, C.: "Prison Population Growth and Crime Reduction", en Journal of Quantitative Criminology, vol. 10 (1994.), $\mathrm{N}^{\circ} 2$, pp. 109-40.

${ }^{19}$ Cfr. Imrohoroglu, A., Merlo, A. y Peter, R.: "What Accounts for the Decline in Crime?", en PIER Working Paper No 01-012; and USC Finance E Business Econ. Working Paper No 01-15. (2001).

${ }^{20}$ Cfr. Grogger, J. and Willis, M.: "The Emergence of Crack Cocaine and the Rise in Urban Crime Rates", en Review of Economics and Statistics, vol. 82 (2000), Noviembre, pp. 519-29.

${ }^{21}$ Cfr. Levitt, S. y Donohue, J.: "The impact of legalized abortion on Crime", en Quarterly Journal of Economics, vol. 116 (2001), Mayo, pp. 379-420.

${ }^{22}$ Comisión de Políticas Criminales del Ministerio Público, presentación sin fecha, entregada al autor por su Coordinador, Jorge Vio D.
} 
de delitos en un tiempo y momento determinados, costo que, en tanto medida de la capaicidad de disuasión del sistema penal, influye en la mayor o menor probabilidad de que esos delitos se cometan.

El modelo considera la aplicación efectiva de penas y el tiempo de duración de éstas, como costos que se toman en cuenta a la hora de decidir involucrarse en una actividad criminal. Según este modelo, existe mayor probabilidad de inmiscuirse en una actividad ilícita cuando es menor el costo de hacerlo que de integrarse a actividades legales. Puesto que el costo de integrarse a las actividades legales está regulado por la situación económica del país y el capital social que haya acumulado la persona, no está al alcance del Ministerio Público diseñar políticas para su control, y por tanto, para efectos de este análisis, debe considerarse una constante. En cambio, el costo probable del delito, que es una función del tiempo y esfuerzo dedicado a su comisión y de la sanción esperada, sí puede (y debiera) ser considerado en la política de persecución penal del Ministerio Público, dado que la sanción esperada es una función compuesta por la probabilidad de ser detenido por el delito, la razón que existe entre el número de detenidos y el de condenados por esa clase de delitos (mientras más se acerca esta razón a 1 , más se aumenta el costo del delito), y la clase y duración de la sanción que efectivamente se aplica (mientras más tiempo duran efectivamente las penas privativas de libertad impuestas, más aumenta el costo del delito). Son estos dos últimos aspectos los que el Ministerio Público puede objetivar al establecer las metas de desempeño y otros estímulos, como una forma de diseñar una política de persecución penal orientada al propósito de reducir la actividad criminal.

Antes de seguir, quisiera hacer notar que el modelo descrito, por "economicista" que parezca, no es más que, como el propio G. Becker señaló ${ }^{23}$, una modernización, en términos matemáticos y econométricos, de los planteamientos de Beccaria, de quien es la afirmación de que "no es la dureza de las penas uno de los más grandes frenos de los delitos, sino la infalibilidad de ellas”, ${ }^{24}$ por una parte; y, por la otra, de Bentham, para quien "el valor de la pena no debe ser menor en ningún caso que el que es suficiente para compensar el beneficio del delito". ${ }^{25}$

Por otro lado, si como se afirma por todos los estudios, la mayor parte de los delitos se comete por un grupo determinado de personas $^{26}$ a una edad determinada (entre $18 \mathrm{y}$

${ }^{23}$ Becker, G., "Crime and Punishment: An Economic Approach", en Journal of Political Economy, vol. 76 (1968), p. 209. Así lo reconoce también Silva Sánchez, J. M.: "Eficiencia y Derecho Penal”, en Anuario de Derecho Penal y Ciencias Penales, 1996, p. 94.

${ }^{24}$ Beccaria, C.: De los delitos y de las penas, Cap. 27 (trad. de J.A. de las Casas), Alianza ed., Madrid, 1990 , p. 71

${ }^{25}$ Cit. por Moreso, J. J.: La teoría del Derecho de Bentham, Barcelona 1992, p. 351, donde aparece, precisamente, en un capítulo que se denomina "análisis económico del Derecho", y se vincula específicamente con las tesis de Becker y Posner.

${ }^{26}$ Cooper, D.: "Nichos Etiológicos y Características de la Delincuencia Urbana y Rural Mapuche y No-Mapuche en Chile”, en http://rehue.csociales.uchile.cl (2000), visitado el 10 de enero de 2006. 
40 años), ${ }^{27}$ mientras más tiempo personas de ese grupo se encuentren cumpliendo penas privativas de libertad, menos delitos podrán cometer. Este efecto de incapacitación es una externalidad positiva de las penas privativas de libertad que una política de persecución criminal orientada a la reducción del delito podría considerar, favoreciendo la obtención de penas efectivas de prisión y de la mayor duración posible dentro de los límites legales. Sin embargo, aquí es necesario tener en cuenta también las externalidades negativas de las penas privativas de libertad, en tanto que su ejecución no es gratuita para la comunidad (deben proveerse cárceles, gendarmes, alimentación, etc. ${ }^{28} \mathrm{y}$, además, genera en los recluidos fenómenos de desocialización importantes que, cuando son liberados, actúan aumentando sus probabilidades de volver a delinquir. ${ }^{29}$ Por lo mismo, en la fijación de metas de desempeño en relación a este aspecto debiera tomar cuidadosamente en cuenta el verdadero beneficio de largas penas privativas de libertad, del que debe descontarse los daños sociales que de ellas se derivan, los cuales parecen conducir a que, marginalmente, el beneficio sea mucho menos relevante que el de otras medidas, como aumentar el número de policías disponibles en tareas de prevención situacional. ${ }^{30}$

El análisis anterior también puede hacerse extensivo a las medidas cautelares, pues la prisión preventiva tiene, desde este punto de vista, los mismos efectos: incapacita para cometer delitos durante su ejecución, y su propia ejecución desocializa y significa importantes gastos fiscales, con la diferencia de que en tales casos no se ha constatado la culpabilidad del autor, por lo que los costos de desocialización son mayores, por la mayor probabilidad de que recaigan en personas que antes no estaban dispuestas a cometer delitos y podrían haber estado sujetas a otras medidas cautelares menos costosas. Luego, para reducir los costos, parece aconsejable propiciar la ejecución de medidas con similares efectos incapacitantes pero menos costosas socialmente (como la vigilancia electrónica), y en caso de ser necesaria la prisión preventiva, reducir sus efectos desocializantes, reduciendo el tiempo de su ejecución, sea mediante el recurso a los juicios inmediatos, sea mediante investigaciones lo más breves posibles.

Finalmente, cabe señalar que en el Documento del año 2004, sobre "Política Nacional de Seguridad Ciudadana", 31 se mencionaba al Ministerio Público como actor relevante en los objetivos de aumentar la proporción de delitos resueltos, y desarrollar estrategias de

${ }^{27}$ Núñez et al, op. cit. Según el Anuario Estadístico Interinstitucional del año 2004 (Tabla 156, p. 193), durante ese período, el $69 \%$ de los delitos se cometieron por personas de entre 18 a 45 años, y un $92 \%$ del total de imputados eran hombres (Tabla 180, p. 217).

${ }^{28}$ Dato que Becker, op. cit., p. 193, toma en cuenta para preferir multas proporcionadas al costo del delito, "whenever feasible".

${ }^{29}$ Las tasas de reincidencia al año 1999 de los condenados recluidos en comparación con los de quienes son beneficiados por medidas alternativas hablan por sí solas de este fenómeno: 47,3\% vs. 16,1\% de los beneficiados con Libertad Vigilada, 20,2\% con reclusión nocturna y 6,4\% con remisión condicional (Fuente: Hofer, M.: "20 años de aplicación de la Ley $\mathrm{N}^{\circ}$ 18.216", en Boletín Jurídico del Ministerio de Justicia, No 4-5 (2003), pp. 157-203, Cuadro $\mathrm{N}^{\circ} 16$ (p. 185)).

${ }^{30}$ Levitt, Why... cit., p. 179.

${ }^{31}$ División de Seguridad Ciudadana: "Política Nacional de Seguridad Ciudadana", Santiago 2004, pp. 44 y 46 . 
persecución focalizadas en tipos de delitos, lugares o hechos reiterados, lo que es coincidente con lo hasta aquí expuesto, con la sola salvedad que aquí se consideran vinculados también a la prevención del delito y no sólo a su control, como en el documento citado. Y en particular, respecto de la formulación de estrategias focalizadas de persecución penal, cuando son territorializadas, siempre se debe considerar que -como ya hemos dicho-sus efectos podrían llevar únicamente al desplazamiento de la actividad criminal a otros territorios y a competencias entre las diferentes Fiscalías Regionales que no necesariamente producirán el mejor resultado social esperable. Además, en el citado documento, se mencionan otros ámbitos donde la actuación del Ministerio Público incidiría en la prevención y control del delito, que no me es posible desarrollar aquí, dado que o bien se comprenden en los anteriores o no corresponden propiamente a la política de persecución penal del Ministerio Público, a saber: en cuanto a prevención social, participar en las intervenciones en barrios vulnerables (aunque no se identifica de qué forma); en lo que respecta a prevención situacional, perfeccionando la atención de víctimas; y en las tareas de control, se le asigna un rol no especificado en las labores de formación para la modernización policial; y en la implementación del nuevo sistema de justicia penal juvenil. ${ }^{32}$

\section{LAS LIMITACIONES A LA FACULTAD DE SUGERIR POLÍTICAS PÚBLICAS QUE AFECTAN O DEBEN IMPLEMENTAR OTRAS INSTITUCIONES}

\section{a) El carácter crítico que conlleva sugerir mejoramientos a otras instituciones}

Como cuando en el Mensaje del CPP 2000 se afirmaba que el sistema penal inquisitivo que antes nos regía estaba "colapsado", toda política pública de mejoramiento supone un diagnóstico en que, explícita o implícitamente, se señala que las instituciones que debieran mejorar no están cumpliendo a cabalidad con su parte en una tarea común (la "efectiva persecución de los delitos, protección de las víctimas y de los testigos, y el adecuado resguardo de los derechos de las personas"), sea por falta de medios materiales o personales, deficiencias de quienes deben ejecutar las labores que se pretenden mejorar o limitaciones de carácter legal para un mejor funcionamiento del conjunto del sistema penal.

En ese escenario, y tal como sucedió con la Reforma Procesal Penal, perfectamente quienes dirigen las instituciones eventualmente aludidas podrían sentirse habilitados para rechazar públicamente tales sugerencias, argumentando que no existen las deficiencias que se sugieren mejorar, que bastaba con que el Ministerio Público actuara coordinadamente con ellas (y que para esos están, por ejemplo, los Planes Regionales Antidelincuencia y sus Comités formados por representantes de la mayoría de las instituciones que forman parte del sistema penal), e incluso, que las deficiencias que fundarían las críticas serían atribuibles a los propios fiscales del Ministerio Público, por desconocimiento de las

\footnotetext{
${ }^{32}$ Id., pp. 38, 41 y 47.
} 
instituciones aludidas y su funcionamiento o su propia impericia. A ello se debe agregar que, conforme a la "Política Nacional de Seguridad Ciudadana" y al Programa de Gobierno de la recién electa Presidenta, se creará un Ministerio de Seguridad Pública, con la finalidad de asumir la dirección superior en estas materias, ${ }^{33}$ y que, por lo mismo, podría estimar que las sugerencias autónomas del Fiscal Nacional podrían derivar en duplicidades y contradicciones innecesarias.

\section{b) La necesidad de fundamentar técnicamente las sugerencias de políticas públicas a implementar por otras instituciones}

Ya hemos señalado que para realizar sugerencias de políticas públicas para la represión y prevención del delito a otras instituciones, es necesario primero que el Ministerio Público fije explícitamente su política en la materia. Ello supone no sólo la elaboración de un documento que recopile y sistematice las políticas de persecución penal que actualmente se están implementando, sino, además, el estudio de los factores que llevan a la comisión de delitos y el diseño de los estímulos institucionales que deben disponerse para que los Fiscales del Ministerio Público logren los nuevos objetivos propuestos, junto con un detallado análisis de costos (directos y alternativos) y beneficios.

Pero también se requiere estudiar la naturaleza y funciones de las instituciones a que tales sugerencias se dirigen, de los recursos de que disponen y los que necesitarían para implementarlas, y del modo cómo su funcionamiento influye en los factores que determinan las probabilidades de comisión de delitos, y de los costos (directos y alternativos) y beneficios de las mejoras que se proponen.

\section{c) El carácter "neutro" de las propuestas de modificación en materia penal y procesal penal}

Los documentos de la Comisión de Políticas Criminales del Ministerio Público enfatizan en el carácter "neutro" de las sugerencias de modificaciones legales que puede hacer el Fiscal Nacional. Sin embargo, parece que en el ámbito de lo penal las modificaciones legales que se propongan difícilmente podrían considerarse "neutrales", a no ser que tuvieran por objeto únicamente la corrección de problemas de redacción o técnicos de una determinada norma, que hagan imposible su cumplimiento o inalcanzables los objetivos que el legislador se propuso. Pero para ello se debe tener precisa y clara noción de los objetivos del legislador, cosa no siempre al alcance de los operadores jurídicos.

Para poner ejemplos: ¿Podrá sugerirse la reinstauración de los delitos de vagancia y mendicidad, sobre la base de estudios que indiquen que entre vagos y mendigos se encuentran más personas dispuestas a cometer delitos que en otros grupos sociales, sin entrar derechamente a la "arena política"?; ¿Podrá sugerirse la eliminación de la audiencia de preparación del juicio oral sin que con ello parezca que se está favoreciendo la

\footnotetext{
${ }^{33}$ División de Seguridad Ciudadana, op. cit., p. 52.
} 
presentación de pruebas por parte de la fiscalía? Pero incluso si se presentase una sugerencia técnica y financieramente fundada para hacer operativas las medidas del Art. 155 CPP, sobre la base de dispositivos de vigilancia electrónica, que tenga en cuenta los mayores costos alternativos de mantener personas no condenadas en prisión preventiva, difícilmente la comunidad va a considerar "neutral" la decisión de adoptar o no tales dispositivos en el sistema penal, tanto por su propia naturaleza como si se consideran una verdadera alternativa a la prisión preventiva. Lo mismo sucederá si las sugerencias se refieren a medidas destinadas a la reinserción de los condenados o a las condiciones de su encierro. En qué medida se considere la reinserción de los condenados un objetivo socialmente deseable y cuántos recursos se destinen a ello parecen ser cuestiones de alta política y no necesariamente "neutrales".

\section{La ACTUAl propuesta de política CRiminal del Ministerio Público}

Sobre la base de lo antes señalado, analizaremos críticamente las propuestas de políticas públicas para la prevención y control del delito contenidas en la Cuenta Pública del año 2006:

\section{a) Propuestas dirigidas a los Poderes Ejecutivo y Legislativo:}

“1.- En cuanto al mejoramiento del sistema procesal penal, perfeccionar la salida alternativa de la suspensión condicional del procedimiento, a través de mecanismos legales y reglamentarios que beneficien el control del cumplimiento de las condiciones fijadas y la reinserción laboral del imputado, contribuyendo de esta manera a su efectiva rehabilitación e impidiendo que vuelva a incurrir en hechos presumiblemente delictuosos".

“2.- Mejorar el sistema legal de control de las medidas cautelares y el cumplimiento alternativo de penas privativas de libertad. Hay que efectuar todas las modificaciones legales y reglamentarias para mejorar significativamente el control de las personas sujetas a medidas cautelares que importan un cierto grado de libertad (tales como permiso de salida durante el día en el caso de la prisión preventiva, arresto domiciliario, prohibición de frecuentar ciertos lugares, etc.) o de cumplimiento alternativo de penas privativas de libertad (remisión condicional, reclusión nocturna o libertad vigilada), e incluso de las actividades que realizan los condenados al interior de los establecimientos penales".

"10.- Apoyar la creación del Ministerio de Seguridad Pública, a fin de que se realice adecuadamente una coordinación de ambas policías y de todos los organismos del Estado, en materias de prevención del delito, sin perjuicio de la coordinación con las labores investigativas y el ejercicio de la acción que realiza en forma autónoma el Ministerio Público".

En primer lugar, estas propuestas adolecen principalmente del grave problema de encontrarse dirigidas a órganos cuya actuación no está bajo el control del Fiscal Nacional, 
quien carece de iniciativa legal y no parece ser posible ni mucho menos deseable que tenga la capacidad política de determinar la agenda o las prioridades legislativas. Además, considerando únicamente el contenido de las propuestas, su generalidad puede considerarse más una declaración de buenas intenciones que una propuesta de regulación, al faltar en ellas propuestas de regulación concretas con una estimación de su incidencia presupuestaria, especialmente necesaria cuando se trata de establecer mecanismos de control que han de requerir destinar recursos y medios para hacerlos efectivos (propuestas 1 y 2).

En segundo término, las propuestas 1 y 2 no definen el rol del Ministerio Público en su eventual implementación. Así, respecto del otorgamiento de la suspensión condicional del procedimiento, al proponer un perfeccionamiento de los mecanismos de control, ¿significará quizás que esta salida alternativa será favorecida por los fiscales adjuntos? ¿respecto a cuáles imputados y a qué clase de delitos? Además, la propuesta parece asumir que las condiciones impuestas en los casos de suspensión condicional del procedimiento tienen efectos rehabilitadores, lo que indicaría que la política del Ministerio Público sería favorecer más esta clase de término del procedimiento que en la medida que se usa actualmente. Sin embargo, si de lo que se trata es de prevenir la comisión de delitos y no la congestión de los tribunales, es necesario estudiar empíricamente el verdadero efecto rehabilitador que se les supone a estas medidas, antes de tomar una decisión de política criminal al respecto.

\section{b) Propuestas referidas al control del cumplimiento de sanciones y medidas alternativas al cumplimiento de las penas privativas de libertad:}

“3.- Cumplimiento efectivo de la pena de multa. Es necesario controlar su cumplimiento efectivo de forma tal que, si el condenado carece de medios para pagarla, sea conmutada por la realización de trabajos en beneficio de la comunidad. Para ello, se requiere de una infraestructura apropiada que, junto con permitir el efectivo cumplimiento, revista de seriedad la amenaza penal asociada a toda conducta ilícita”.

“4.- Apoyo del Ministerio Público a la generación de una red social que funcione coordinadamente con organismos públicos y privados para ofrecer capacitación laboral, tanto bajo la suspensión condicional como bajo la ley 18.216. El desarrollo técnico de esta política demandará inversiones y recursos destinados a la reinserción laboral y a la construcción de una red de coordinación entre organismos públicos y privados que apoyen la rehabilitación del beneficiado”.

Ambas propuestas apuntan a establecer, en general, redes que permitan el cumplimiento de penas sustitutivas a la multa y medidas alternativas a las privativas de libertad, a través de formas de capacitación laboral. Lamentablemente, el loable propósito de estas propuestas parece bastante poco concretizado: ¿a qué instituciones "públicas” y "privadas” se refieren?, ¿cuál es el compromiso del Ministerio Público al respecto, esto es, cuántos convenios suscribirá que representen qué cantidad de personas en condiciones 
de cumplir con trabajos o capacitación laboral? Nada de esto se explicita. Mucho menos, cuántas plazas traducidas en horas-hombre de capacitación se requieren efectivamente. Y lo más importante: ¿Se encontrarán entre las metas de los fiscales adjuntos Ministerio Público este año hacer efectivo el control del pago de las multas y su sustitución por la privación de libertad cuando corresponde, así como el efectivo cumplimiento de las condiciones en que se otorgan los beneficios de la Ley $\mathrm{N}^{\circ}$ 18.216? ¿Qué proporción de cumplimiento efectivo se considera un éxito en la gestión?

\section{c) Propuestas referidas a la persecución y prevención de los delitos}

“5.- En cuanto a las medidas para una más efectiva persecución de los delitos, se propone fomentar la transferencia de información, con ayuda de medios tecnológicos, entre los sistemas informáticos de las policías y el Ministerio Público, y el acceso a las bases de datos y bancos de imágenes públicas y privadas (Policía, Registro Civil, Gendarmería, grandes tiendas, supermercados, etc.)”.

“6.- Potenciamiento y actualización de las cámaras de vigilancia en los espacios públicos, por parte de las municipalidades e intendencias y su inserción dentro de las estrategias antidelictuales coordinadas entre el Ministerio Público y las policías”.

“7.- Potenciamiento de la capacidad para interceptación telefónica y registro de tráficos telefónicos, tanto en las compañías de teléfonos como en las policías”.

"8.- Intensificación de campañas mediáticas destinadas a difundir hábitos y mejores prácticas de prevención del delito. Estas campañas deben cambiar malos hábitos fuertemente arraigados en la población, tales como el robo en supermercados y tiendas de autoservicio o la conducción en estado de ebriedad o bajo la influencia del alcohol o drogas, o la compra de artículos, tanto por la vía de mostrar los efectos indeseados que tales conductas puedan acarrear a los propios hechores como también del llamado dirigido a quienes sean testigos de la comisión de esos delitos para que los denuncien sin temor.

9.- Generar instancias de coordinación entre el Ministerio Público, el Gobierno, las Policías, las municipalidades y el sector privado con la finalidad de individualizar, mediante mapas, los mercados de reducción de especies robadas y diseñar estrategias de intervención de dichos mercados. De esta manera, los fiscales podrán perseguir decididamente el delito de receptación, extendiendo su acción a los mercados ilícitos de reducción de especies.

En cuanto a las medidas de Coordinación que se proponen, la cuestión radica en determinar cuántos recursos propios invertirá el Ministerio Público en ellas y qué resultados se esperan de esa inversión. ¿Traspasará el Ministerio Público parte de sus fondos de inversión a las otras instituciones que señalan las propuestas 5 y 9, para cumplir el 
objetivo de establecer un mecanismo de transferencia de información? ¿Se establecerán convenios específicos al respecto? ¿Cuándo estaría en operaciones el sistema? ¿Se destinará personal del Ministerio Público específicamente a las labores de coordinación? ¿Cuánto significa esta inversión? ¿Hay una meta que indique si los esfuerzos han sido o no exitosos? En cuanto a las medidas 6, 7 y 9, en lo que involucran inversiones de otras instituciones: ¿cuánto se espera que ello sea?, ¿si los municipios carecen de recursos, pondrá cámaras el Ministerio Público?, ¿cuánto cuesta este programa? Y en cuanto a la propuesta 8: ¿La campaña "mediática” la dirigirá y financiará el Ministerio Público o se espera que otros organismos lo hagan? Si es el primer caso: ¿cómo se medirán los beneficios esperados, esto es, cuál es la meta de reducción de las clases de delitos a que se refieren y de aumento de las "denuncias sin temor"? Y en general, respecto a todas estas propuestas, y en la medida que signifiquen recursos humanos y materiales del Ministerio Público: ¿cuál es su costo alternativo respecto a las funciones constitucionales que se entregan al Ministerio Público, esto es, investigar los delitos y sostener la acción penal? Finalmente, quisiera llamar la atención respecto de la forma como se presenta la propuesta 9, pues si por ley todas las denuncias terminan ingresándose al Ministerio Público y éste ya dispone de sistemas informáticos avanzados y se ha implementado el georeferenciamiento en algunas ciudades, como se destaca en esta Cuenta Pública del año 2006 (p. 5s), ¿por qué supeditar la extensión de esta medida a la colaboración con otros organismos cuando es posible adoptar esta medida como propia, si ha sido eventualmente exitosa?

\section{Resumen}

En síntesis: mi opinión es que la mejor forma que tiene el Ministerio Público de asumir la oportunidad que significa la facultad de sugerir políticas públicas en materias de prevención y represión del delito, es explicitar y fijar su propia política de persecución criminal, orientándola hacia el objetivo de reducir la actividad delictiva, de manera que, sobre la base del conocimiento aportado por la investigación criminológica con fundamento científico y empírico, se establezcan las estrategias locales, regionales y nacionales para cumplir esos objetivos, junto con los estímulos institucionales necesarios para que en su actuación diaria los Fiscales del Ministerio Público trabajen para el logro de ese objetivo. Al menos, ello supone un trabajo conjunto de esta Comisión con las encargadas de la implementación del art. 17 a) LOCMP, pues tales estrategias y estímulos necesariamente han de reflejarse en instrucciones de carácter general y en las correspondientes metas de desempeño de cada Fiscalía Regional.

Sólo una vez hecho lo anterior cabría sugerir a otras instituciones medidas de colaboración con esa política de persecución criminal, pero siempre tomando en cuenta que toda sugerencia de mejoramiento supone una crítica, y que difícilmente en estas materias podrá considerarse "neutral" la proposición de una modificación legal. De otro modo, el resultado no puede ser distinto a las propuestas criticadas en el apartado anterior, donde no se define con claridad qué hará el Ministerio Público para prevenir y controlar 
la actividad delictual, cuánto invertirá en ello, cuál es el costo alternativo asociado y cómo medirá sus resultados, aparte, claro, de no hacerse mención alguna de qué manera, mejorando la gestión de sus funciones constitucionales (investigar los delitos y sostener la acción penal), contribuirá al objetivo de prevenir y controlar la criminalidad.

\section{BIBLIOGRAFÍA}

Beccaria, C., De los delitos y de las penas, Cap. 27 (trad. de J.A. de las Casas), Alianza ed., Madrid, 1990, p. 71

Becker, G., "Crime and Punishment: An Economic Approach", en Journal of Political Economy, vol. 76 (1968), p. 209.

Blumstein, A. y Rosenfeld, R., "Explaining Recent Trends in U.S. Homicide Rates", en Journal of Criminal Law and Criminology, vol. 88 (1998), No 4, p. 1179.

Comisión de Política Criminal del Ministerio Público, Minuta Sobre el alcance de la modificación introducida por la Ley 20.074 al Art. 21 de la Ley Orgánica del Ministerio Público, Noviembre de 2005, no publicada.

Cooper, D., "Nichos Etiológicos y Características de la Delincuencia Urbana y Rural Mapuche y No-Mapuche en Chile", en http://rehue.csociales.uchile.cl (2000), visitado el 10 de enero de 2006.

De la Puente, P. y Torres, E., "Seguridad ciudadana y prevención del delito. Un análisis crítico de los modelos y estrategias contra la criminalidad”, en Estudios Criminológicos y Penitenciarios, $\mathrm{N}^{\circ} 1$ (2000), pp. 15-62.

División de Seguridad Ciudadana, "Política Nacional de Seguridad Ciudadana", Santiago 2004, pp. 44 y 46.

El Mercurio, Cuerpo C, Sábado 21 de enero de 2006.

Grogger, J. AND Willis, M., "The Emergence of Crack Cocaine and the Rise in Urban Crime Rates", en Review of Economics and Statistics, vol. 82 (2000), Noviembre, pp. 519-29.

Imrohoroglu, A., Merlo, A. y Peter, R., "What Accounts for the Decline in Crime?", en PIER Working Paper $\mathrm{N}^{\circ}$ 01-012; and USC Finance E Business Econ. Working Paper $\mathrm{N}^{\circ}$ 01-15. (2001).

Kennedy, D. et al, "Youth Violence in Boston: Gun Markets, Serious Youth offenders, and a Use-reduction strategy", en Law and Contemporary Problems, vol. 59 (1996), $\mathrm{N}^{\circ} 1$, pp. 147-195.

Hofer, M., “20 años de aplicación de la Ley No 18.216”, en Boletín Jurídico del Ministerio de Justicia, No 4-5 (2003), pp. 157-203.

Langan, P., “America's Soaring Prison Population”, en Science 251 (1991), marzo, pp. $1568-1573$.

LevitT, S., "Understanding Why Crime Fell in the 1990s: Four Factors that Explain the Decline and Six that Do Not", en Journal of Economics Perspectives, vol. 18 (2004), No 1, pp. 163-190. Su traducción puede verse en Ius et Praxis, vol. 10 (2004) N 2, pp. 97-137, traductor: Matus, J. P.

LevitT, S. y Donohue, J., "The impact of legalized abortion on Crime", en Quarterly Journal of Economics, vol. 116 (2001), Mayo, pp. 379-420.

LotT, J. y Mustard, D., "Right-to-Carry Concealed Guns and the Importance of Deterrence", en Journal of Legal Studies, vol. 26 (1997), Enero, pp. 1-68.

Marvell, T. y Moody, C., "Specification Problems, Police Levels, and Crime Rates", en Criminology, vol. 34 (1996.), Noviembre, pp. 609-46. 
Marvell, T. y Moody, C., "Prison Population Growth and Crime Reduction", en Journal of Quantitative Criminology, vol. 10 (1994), N², pp. 109-40.

Matus, J. P., “¿Por qué no bajan las tasas de criminalidad en Chile?”, en Revista de Derecho de la Pontificia Universidad Católica de Valparaíso, vol. XXVI (2005), No 1, pp. 67-92.

Matus, J.P., "Criterios de actuación del Ministerio Público en materias penales. Recensión del texto 'Reforma procesal penal. Oficios del Fiscal Nacional. Materias penales'. 2001-2004. Santiago 2005”, en Ius et Praxis, vol 11 (2005) N 2, pp. 353-360.

Moreso, J. J., La teoría del Derecho de Bentham, PPU, Barcelona, 1992.

NúÑez, J., Rivera, J., Villavicencio, X. y Molina, Ó., "Determinantes socioeconómicos y demográficos del crimen en Chile: evidencia desde un panel de datos de las regiones chilenas", en Estudios de Economía, vol. 30 (2003), Nº 1, pp. 55-85.

Piedrabuena, G., "Quinta Cuenta Pública de Actividades del Ministerio Público, Efectuada por el Fiscal Nacional, don Guillermo Piedrabuena Richard (Art. 21 de la Ley 19.640), de 26 de abril de 2004", en http://www.ministeriopublico.cl/index.asp, visitado el 10 de enero de 2006

Piedrabuena, G., "Sexta Cuenta Pública de Actividades del Ministerio Público, Efectuada por el Fiscal Nacional, don Guillermo Piedrabuena Richard (Art. 21 de la Ley 19.640), de 26 de abril de 2005", en http://www.ministeriopublico.cl/index.asp, visitado el 2 de mayo de 2006, p. 8s.

Piedrabuena, G., "Séptima Cuenta Pública del Ministerio Público, Efectuada por el Fiscal Nacional, don Guillermo Piedrabuena (Art. 21 de la Ley 19.640), 28 de abril de 2006”, en http://www.ministeriopublico.cl/index.asp, visitado el 20 de mayo de 2006.

Silva SÁnchez, J. M., "Eficiencia y Derecho Penal", en Anuario de Derecho Penal y Ciencias Penales, 1996, p. 94. 